\title{
Vortex Thermometry for Turbulent Two-Dimensional Fluids
}

\author{
Andrew J. Groszek, ${ }^{1}$ Matthew J. Davis, ${ }^{2}$ David M. Paganin, ${ }^{1}$ Kristian Helmerson, ${ }^{1}$ and Tapio P. Simula ${ }^{1}$ \\ ${ }^{1}$ School of Physics and Astronomy, Monash University, Victoria 3800, Australia \\ ${ }^{2}$ School of Mathematics and Physics, University of Queensland, Queensland 4072, Australia
}

(Received 17 February 2017; published 19 January 2018)

\begin{abstract}
We introduce a new method of statistical analysis to characterize the dynamics of turbulent fluids in two dimensions. We establish that, in equilibrium, the vortex distributions can be uniquely connected to the temperature of the vortex gas, and we apply this vortex thermometry to characterize simulations of decaying superfluid turbulence. We confirm the hypothesis of vortex evaporative heating leading to Onsager vortices proposed in Phys. Rev. Lett. 113, 165302 (2014), and we find previously unidentified vortex power-law distributions that emerge from the dynamics.
\end{abstract}

DOI: 10.1103/PhysRevLett.120.034504

Turbulence arises in chaotic dynamical systems across all scales, from mammalian cardiovascular systems, to climate, and even to the formation of stars and galaxies [1]. The unpredictability inherent to turbulent systems is further confounded by physical properties such as boundaries and spatial dimensionality, and because of its complexity, there is currently no unified theoretical framework to explain turbulence. As such, there is a need to develop new methods to characterize the evolution of turbulent states in order to provide further insights into this important problem.

Onsager developed a model of statistical hydrodynamics to describe turbulence in two-dimensional (2D) flows [2]. In this representation, the fluid is modeled by an $N$-particle gas of interacting pointlike vortices which can be characterized by an equilibrium temperature. As the bounded system of vortices has a finite configuration space, the entropy $S$ of the system has a maximum, and hence there is a range of energy $E$ where the absolute Boltzmann temperature $T=(\partial S / \partial E)^{-1}$ becomes negative [2-4]. These states correspond to large-scale rotational flows known as Onsager vortices [2].

In driven 2D incompressible fluids, negative temperature Onsager states are known to emerge dynamically, effectively giving rise to order from chaos. This peculiar phenomenon is understood to be associated with an inverse energy cascade, in which energy flows towards the largest length scales in the system $[5,6]$. However, it is not clear whether this process should carry over to the regime of superfluid turbulence due to the compressibility of the superflow [7]. Despite this, many recent theoretical works involving superfluid Bose-Einstein condensates (BECs) have suggested that large-scale, same-sign, Onsager vortex clusters play an important role in $2 \mathrm{D}$ quantum turbulence [8-17]. In superfluids, much of the focus to date has been on decaying-rather than driven-turbulence [9,11-14, 17-24] since this scenario removes the complications of stirring. A variety of concepts have been applied to the problem, including holographic duality $[25,26]$ and nonthermal fixed points [27-29].

Experimentally, BECs provide unprecedented opportunities to investigate 2D superfluid turbulence due to the high degree of controllability available in these systems. It is now possible to create and image complex vortex configurations such as dipoles [30-33], few-vortex clusters [34], and quantum von Kármán vortex streets [35]. Many experiments have also been devoted to the study of quantum turbulence in both two-dimensional $[11,19,20,23]$ and three-dimensional $[22,36,37]$ geometries. However, the formation of Onsager vortex structures in statistical equilibrium has not yet been reported. Recent theoretical works have suggested that one significant obstacle is the harmonic trapping commonly used in experiments, as vortex clusters appear to be suppressed in this geometry $[14,20,38]$. In addition, the detection of vortex circulation signs is experimentally difficult, and only recently have techniques been proposed [39] and implemented [23] to achieve this. Analysis of turbulent dynamics is made even more challenging by current condensate imaging methods, which only allow a small number of frames to be captured for a single experimental realization [40]. As such, it is desirable to be able to characterize the state of a turbulent fluid using a robust method of statistical analysis that links the instantaneous microscopic configuration of the system to its macroscopic behavior. Onsager's thermodynamical description of turbulence is one such method, and hence we propose to use its central observable - the vortex temperature-for this purpose. In contrast to velocimetry-based observables that require the measurement of the velocities of the atoms, the thermometry presented here only requires the measurement of the positions and circulation signs of the quantized vortices.

We first outline our method for measuring the temperature of the vortex gas, before examining a specific case of decaying superfluid turbulence using mean-field 
Gross-Pitaevskii simulations. In the dynamics, we observe that the vortex gas undergoes rapid equilibration before settling into a quasiequilibrium state, where it continues to heat adiabatically via vortex evaporation [13]. We have discovered that, in this evolution, the numbers of clusters, dipoles, and free vortices follow robust power laws with respect to the total vortex number. The existence of this quasiequilibrium allows quantitative thermometry of the turbulent fluid.

To calibrate the vortex thermometer, we use Monte Carlo (MC) simulations to map out the equilibrium vortex configurations as a function of the inverse temperature $\beta=1 / k_{B} T$, where $k_{B}$ is Boltzmann's constant. We do this for a system of $N_{v}=50$ point vortices with equal numbers of clockwise and anticlockwise circulations. Other values of $N_{v}$ are considered in Ref. [41]. We use a point vortex Hamiltonian corresponding to a uniform fluid within a circular boundary of radius $R[13,43]$, and set a hard vortex core of radius $0.003 R$ to prevent energy divergences. As we vary the temperature across both positive and negative regimes, we quantify the effect on the vortex configuration using a vortex classification algorithm [10,44]. The algorithm uniquely divides the vortex gas into three separate components: clusters of $\geq 2$ like-sign vortices, closely bound vortex-antivortex dipoles, and relatively isolated free vortices (for further details, see Ref. [44]). We then calculate the number of clusters $N_{c}$, dipoles $N_{d}$, and free vortices $N_{f}$ as functions of temperature, and the resulting fractional population curves are presented in Fig. 1.

At low positive absolute temperatures (left-hand side of Fig. 1), the vortex gas is at its "coldest," as both the energy and entropy are minimized. In this regime, bound vortexantivortex dipole pairs dominate the configuration, as shown in the schematic inset of Fig. 1. Above the Berezinskii-Kosterlitz-Thouless (BKT) critical temperature $\beta_{\text {BKT }}$ [45-49], the vortex dipoles dissociate, causing an increase in both the energy and entropy. At $\beta=0$, the vortex configuration becomes a disordered arrangement of vortices and antivortices, thereby maximizing the entropy. In the negative temperature region, low-entropy clusters of like-sign vortices tend to form (see schematic inset), and because of their high energy, these negative temperature states are "hotter" than those at positive temperature. Above the critical temperature $\beta_{\mathrm{EBC}}$, the vortices form an EinsteinBose condensate (EBC), a state where the Onsager vortex clusters condense, as indicated by the saturation of the cluster population in Fig. 1 [13,44,50]. For a neutral vortex gas the two aforementioned critical temperatures are defined as $\beta_{\mathrm{BKT}}=2 / E_{0}$ and $\beta_{\mathrm{EBC}}=-4 / N_{v} E_{0}$ [6], respectively, where the energy $E_{0}=\rho_{s} \kappa^{2} / 4 \pi$ is defined in terms of the superfluid density $\rho_{s}$ and the quantum of circulation $\kappa=h / m$, with $m$ being the mass of the condensed atoms.

Figure 1 demonstrates that the dipole and cluster populations are monotonic functions of $\beta$-this is the key observation enabling thermometry of the vortex gas.

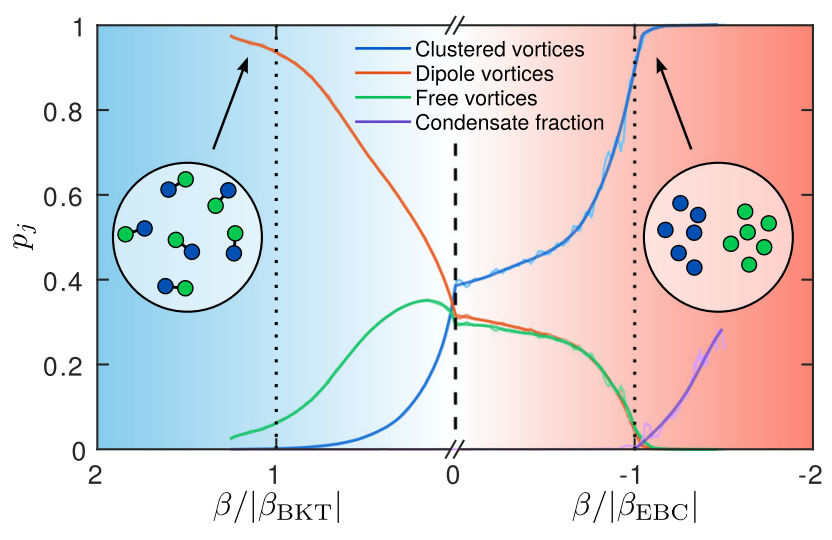

FIG. 1. Fractional population $p_{j}=N_{j} / N_{v}$ of each component of the vortex gas (where $j \in\{c$ : clustered vortices; $d$ : dipole vortices; $f$ : free vortices $\}$ ) and Einstein-Bose condensate fraction (described in text), as functions of inverse temperature $\beta$. The fluctuating faint lines show the raw data, while the smooth dark lines show cubic spline fits. The negative temperature axis is scaled by the critical temperature $\left|\beta_{\mathrm{EBC}}\right|$, and the positive temperature axis by $\left|\beta_{\mathrm{BKT}}\right|$, causing an apparent discontinuity in the slopes at $\beta=0$. The dashed vertical line indicates $\beta=0$, and the dotted vertical lines highlight the two critical temperatures. The shaded background represents the energy of the system (red and blue correspond to "hot" or high energy and "cold" or low energy, respectively). Schematic vortex configurations at each temperature extreme are depicted in the insets, where vortices (antivortices) are shown as blue (green) filled circles.

Given an arbitrary vortex configuration in thermal equilibrium, we may determine its temperature by calculating the populations of clusters and/or dipoles and by comparing the obtained values to the curves in Fig. 1. Strictly speaking, the $p_{j}(\beta)$ curves in the negative temperature region of Fig. 1 are dependent on the vortex number. However, we repeated our MC simulations for $N_{v}=100$ and 200 vortices and found that, for the vortex numbers relevant to the dynamical simulations presented here, there is no qualitative change to the thermometry curves, and the quantitative change is not significant (see Ref. [41]). The cluster and dipole fractions are not the only observables that vary monotonically with vortex temperature in our MC simulations. For example, both the energy and dipole moment of the vortex gas also fulfill this requirement [13] and could therefore, in principle, be used for thermometry. However, of all variables considered, we have found that the cluster and dipole fractions provide the most robust thermometers.

Also shown in Fig. 1 is the Einstein-Bose condensate fraction, which quantifies the density of vortices in the largest cluster (for details, see Ref. [44]). For $\beta>\beta_{\mathrm{EBC}}$, the condensate fraction is zero, but when $\beta<\beta_{\mathrm{EBC}}$, it rises sharply. In this extreme temperature region, the other thermometers saturate and the condensate fraction becomes the relevant observable for vortex thermometry. 
As an application of our vortex thermometer, we use it to characterize decaying turbulence in a disk-shaped BEC as previously studied in Refs. [13,14]. We simulate the two-dimensional, time-dependent Gross-Pitaevskii equation (GPE),

$$
i \hbar \frac{\partial}{\partial t} \psi=\left(-\frac{\hbar^{2}}{2 m} \nabla^{2}+V_{\mathrm{tr}}+g_{2 \mathrm{D}}|\psi|^{2}\right) \psi,
$$

where $\psi \equiv \psi(\mathbf{r}, t)$ is the classical field of the Bose gas and $g_{2 \mathrm{D}}$ is the two-dimensional interaction parameter resulting from the $s$-wave atomic collisions. To obtain the uniform circular geometry, we use a two-dimensional power-law trapping potential of the form $V_{\mathrm{tr}}=\mu(r / R)^{50}$, where $r=$ $\sqrt{x^{2}+y^{2}}$ is the radial distance from the axis of the trap, $\mu$ is the chemical potential, and $R \approx 171 \xi$ is the radius of the trap, measured in units of the healing length $\xi=\sqrt{\hbar^{2} / 2 m \mu}$ [14]. The interaction parameter is set to $g_{2 \mathrm{D}}=4.6 \times$ $10^{4} \hbar^{2} / \mathrm{m}$. We solve the GPE using a fourth-order, splitstep Fourier method on a $1024^{2}$ numerical grid with spacing of approximately $\xi / 2$. Turbulence is generated by imprinting vortices into the phase of $\psi$ and evolving Eq. (1) for a short amount of imaginary time to establish the vortex core structures. We detect vortices and their circulation signs within $r<0.98 R$ by locating singularities in the phase of the field.

The initial vortex configurations used in our GPE simulations are produced by randomly drawing $N_{v}=$ 800 vortex locations from a uniform distribution, with equal numbers of each circulation sign. The resulting state is well approximated to have $\beta \approx 0$, although the short imaginary time propagation step causes a small amount of cooling towards positive temperatures. As the turbulence decays, the vortices annihilate and the vortex gas evaporatively heats, resulting in the emergence of two large Onsager vortices at late times $[13,14]$. Three sample frames from a single simulation are shown in Fig. 2, where panels (a)-(c) show the density $|\psi|^{2}$ of the fluid, and panels (d)-(f) show the corresponding vortex configuration after the vortex detection and classification steps. A Helmholtz decomposition [8] has been used to extract the divergence-free component of the condensate velocity field, and the resulting streamlines are also shown in the lower panels. The Onsager vortex clusters are clearly visible in panel (f).

The number of clusters, dipoles, and free vortices are shown in Fig. 3 as functions of both time $t$ (inset) and the total number of vortices $N_{v}(t)$. The time-dependent populations (inset) do not follow any simple function. However, the populations as functions of the total number of vortices (main frame) show clear power-law scaling behavior. The corresponding power laws are $N_{c} \propto N_{v}^{\alpha}$, $N_{d} \propto N_{v}^{\gamma}, N_{f} \propto N_{v}^{\delta}$, and $N_{v c} \propto N_{v}^{\varepsilon}$, with measured values $\alpha=0.79, \gamma=1.21, \delta=1.18$, and $\varepsilon=-0.25$. These are suggestive of rational value power laws with exponents

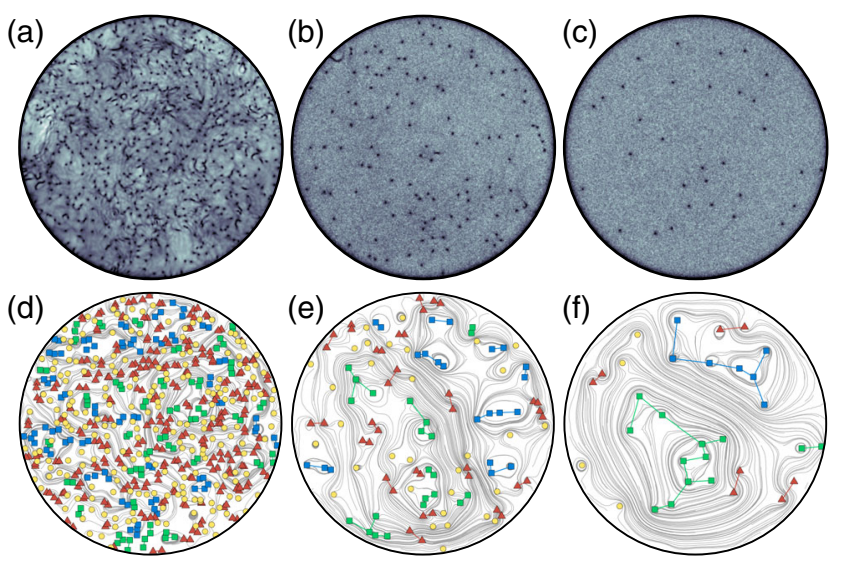

FIG. 2. Freely decaying two-dimensional quantum turbulence. Panels (a)-(c) show the classical field density $|\psi|^{2}$, for respective times $t \approx(25,7500$, and 74000$) \hbar / \mu$, and reveal the vortices as dark spots of zero density. The gray scale in each panel is normalized to the respective peak value of $|\psi|^{2}$. Panels (d)-(f) correspond to panels (a)-(c), respectively, and show the vortices in positive (negative) clusters as blue (green) squares, dipoles as red triangles, and free vortices as yellow circles. Note that each vortex dipole contains one vortex and one antivortex. The streamlines in (d)-(f) are obtained by calculating the incompressible component of the velocity field of the classical field describing the Bose gas.

$\alpha=4 / 5, \gamma=\delta=6 / 5$, and $\varepsilon=-1 / 4$. The mean number of vortices per cluster $N_{v c} \equiv N_{c} / N_{c l}$, where $N_{c l}$ is the total number of clusters of any size at a given time. To study the effects of system size on these power laws, we have also considered two smaller disk-shaped systems of radii

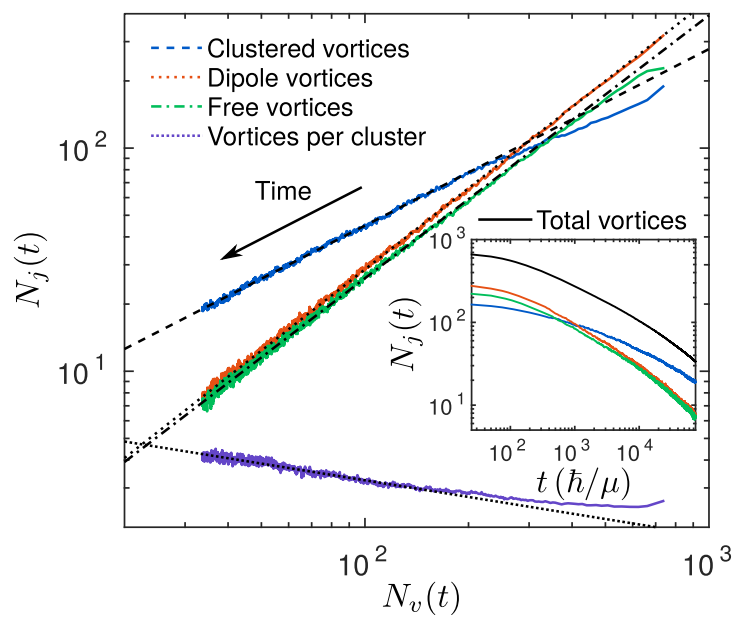

FIG. 3. Decay of the vortex populations $N_{j}(t)$ (where $j \in\{c:$ clustered vortices; $d$ : dipole vortices; $f:$ free vortices $\}$ ) in each component of the vortex gas, and the growth of the number of vortices per cluster $N_{v c}(t)$, as functions of the total vortex number $N_{v}(t)$. The data have been averaged over 80 simulations, with power-law fits shown as straight lines. Note that time flows from right to left in this figure. The inset shows the total number of vortices and the number of vortices in each component of the vortex gas as functions of time. 
$R \approx 49 \xi$ and $R \approx 85 \xi$, respectively, each with $N_{v}=100$ vortices initially imprinted. We find that the scaling behavior is unchanged in these smaller systems, suggesting that the evolution of the vortex gas is underpinned by a universal microscopic process.

In this system, the primary cause of vortex number decay is the annihilation of vortex-antivortex dipoles. Despite this, the populations of dipoles and free vortices follow approximately the same power law, demonstrating an interconversion between the vortex populations. However, a distinct power law emerges for the vortex clusters. This behavior points toward a two-fluid model, where the dipoles and free vortices behave as a weakly interacting thermal cloud, while the clusters act as a quasicondensate whose relative weight grows over time as a result of vortex evaporative heating. Extrapolating the data toward $N_{v} \rightarrow 0$ leads to the inevitable decay of all dipoles and free vortices, with only Onsager vortex clusters remaining. At this point, the rate of pair annihilation becomes insignificant in the dynamics due to the very low probability of vortex-antivortex collisions.

In Fig. 3 , the $N_{d}$ and $N_{f}$ curves are well described by the $N_{v}^{6 / 5}$ scaling throughout the dynamical evolution. The $N_{c}$ curve, on the other hand, only begins to follow the $N_{v}^{4 / 5}$ power law once the total vortex number has decayed to $N_{v} \lesssim 200$, suggesting that the statistical behavior of the vortex gas changes at this point in the dynamics. In accordance with the existence of power-law scaling, we interpret this change to be the realization of a state of quasiequilibrium for the decaying turbulence. Under this quasiequilibrium condition, the vortex evaporative heating process becomes adiabatic in the sense that the vortex gas is able to rearrange into a higher entropy configuration between the vortex annihilation events. For $N_{v} \gtrsim 200$, the vortex number decays too rapidly for this to be possible. This quasiequilibrium condition is not a true equilibrium of the system since vortex-antivortex annihilations and vortexsound interactions are continuously driving energy from the vortices into the sound field. Presumably, the true equilibrium of the condensate will only be realized when all vortices have decayed and the total entropy of the system is maximized. In Ref. [41], we present vortex number decay data for a range of other initial vortex configurations, observing in all cases evidence for the same power-law and quasiequilibration behavior.

We now have an algorithm to assign a vortex temperature to the dynamical GPE simulations. We determine the fractional populations of vortex dipoles and clusters as a function of time and use each of these to read off a temperature from the curves in Fig. 1. The two resulting measurements of $\beta(t)$ are presented in the main frame of Fig. 4. Both measurements show that the temperature of the vortex gas is spontaneously increasing as Onsager vortex clusters form, thereby confirming the evaporative heating scenario posited in Ref. [13]. At late times, a small

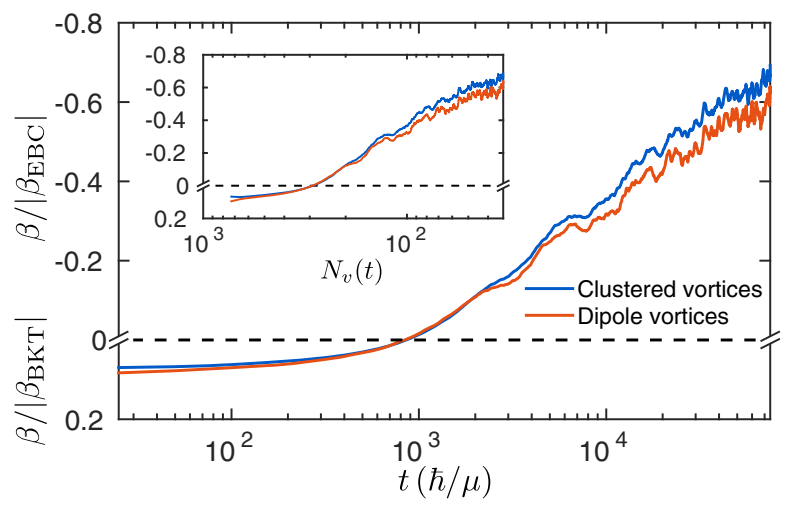

FIG. 4. Inverse temperature of the vortex gas as a function of time, averaged over a set of dynamical GPE simulations. The temperature is measured independently using the populations of both clusters and dipoles. In the inset, the temperature readings from each thermometer are shown as a function of the total vortex number $N_{v}(t)$. As in Fig. 1, the positive and negative temperature regions have been scaled by their respective critical temperatures, and a dashed horizontal line denotes $\beta=0$. The vertical axis of the inset is the same as for the main frame.

discrepancy between the two temperature readings emerges, which we attribute to the compressibility of the fluid not accounted for in the MC model. The same temperature measurements are plotted as a function of the total vortex number in the inset. Based on our quasiequilibrium interpretation discussed above, we note that the temperature reading is strictly only valid for $N_{v} \lesssim$ $200(t \gtrsim 2000 \hbar / \mu)$ since outside of this range the vortices are out of equilibrium and their temperature is not well defined. To obtain these curves, we have applied the thermometer calibrated with $N_{v}=50$ vortices, despite the fact that $N_{v}$ varies between approximately 30 and 200 throughout the equilibrium dynamical evolution. In Ref. [41], we show that using a thermometer calibrated with a different number of vortices does not affect the qualitative shape of the $\beta(t)$ curve.

We have developed a methodology that allows the temperature of point vortices in two-dimensional fluids to be determined using only the information about the vortex positions and their signs of circulation. We have applied the vortex gas thermometers to freely decaying twodimensional quantum turbulent systems and quantitatively shown the transition to negative temperatures and the emergence of Onsager vortices due to the evaporative heating of the vortex gas $[13,14]$. Our vortex thermometers may also be useful for characterization of turbulent classical fluids, as the continuous vorticity distributions can be approximated accurately by a discretized set of point vortices before performing the vortex classification and thermometry. This methodology may therefore open new pathways to quantitative studies of two-dimensional turbulence.

We thank Matthew Reeves for useful feedback on the manuscript. We acknowledge financial support from an 
Australian Government Research Training Program Scholarship (A. G.), the Australian Research Council via Discovery Projects No. DP130102321 (T. S., K. H.), No. DP170104180 (T. S.), No. DP160103311 (M. D.), and the nVidia Research Grant Scheme. This research was undertaken with the assistance of resources from the National Computational Infrastructure (NCI), which is supported by the Australian Federal Government.

[1] P. A. Davidson, Turbulence: An Introduction for Scientists and Engineers (Oxford University Press, New York, 2015).

[2] L. Onsager, Nuovo Cimento 6, 279 (1949).

[3] E. M. Purcell and R. V. Pound, Phys. Rev. 81, 279 (1951).

[4] N. F. Ramsey, Phys. Rev. 103, 20 (1956).

[5] R. H. Kraichnan, Phys. Fluids 10, 1417 (1967).

[6] R. H. Kraichnan and D. Montgomery, Rep. Prog. Phys. 43, 547 (1980).

[7] R. Numasato, M. Tsubota, and V. S. L'vov, Phys. Rev. A 81, 063630 (2010).

[8] A. S. Bradley and B. P. Anderson, Phys. Rev. X 2, 041001 (2012).

[9] A. C. White, C. F. Barenghi, and N. P. Proukakis, Phys. Rev. A 86, 013635 (2012).

[10] M. T. Reeves, T. P. Billam, B. P. Anderson, and A. S. Bradley, Phys. Rev. Lett. 110, 104501 (2013).

[11] T. W. Neely, A. S. Bradley, E. C. Samson, S. J. Rooney, E. M. Wright, K. J. H. Law, R. Carretero-González, P. G. Kevrekidis, M. J. Davis, and B. P. Anderson, Phys. Rev. Lett. 111, 235301 (2013).

[12] T. P. Billam, M. T. Reeves, B. P. Anderson, and A. S. Bradley, Phys. Rev. Lett. 112, 145301 (2014).

[13] T. Simula, M. J. Davis, and K. Helmerson, Phys. Rev. Lett. 113, 165302 (2014).

[14] A. J. Groszek, T. P. Simula, D. M. Paganin, and K. Helmerson, Phys. Rev. A 93, 043614 (2016).

[15] A. Skaugen and L. Angheluta, Phys. Rev. E 93, 032106 (2016).

[16] A. Skaugen and L. Angheluta, Phys. Rev. E 95, 052144 (2017).

[17] H. Salman and D. Maestrini, Phys. Rev. A 94, 043642 (2016).

[18] P. M. Walmsley and A. I. Golov, Phys. Rev. Lett. 100, 245301 (2008).

[19] S. J. Rooney, T. W. Neely, B. P. Anderson, and A. S. Bradley, Phys. Rev. A 88, 063620 (2013).

[20] W. J. Kwon, G. Moon, J. Choi, S. Seo, and Y. Shin, Phys. Rev. A 90, 063627 (2014).

[21] A. Cidrim, F. E. A. dos Santos, L. Galantucci, V. S. Bagnato, and C. F. Barenghi, Phys. Rev. A 93, 033651 (2016).

[22] N. Navon, A. L. Gaunt, R. P. Smith, and Z. Hadzibabic, Nature (London) 539, 72 (2016).

[23] S. W. Seo, B. Ko, J. H. Kim, and Y. Shin, Sci. Rep. 7, 4587 (2017).
[24] P. M. Walmsley and A. I. Golov, Phys. Rev. Lett. 118, 134501 (2017).

[25] P. M. Chesler, H. Liu, and A. Adams, Science 341, 368 (2013).

[26] Y. Du, C. Niu, Y. Tian, and H. Zhang, J. High Energy Phys. 12 (2015) 018.

[27] B. Nowak, D. Sexty, and T. Gasenzer, Phys. Rev. B 84, 020506 (2011).

[28] B. Nowak, J. Schole, D. Sexty, and T. Gasenzer, Phys. Rev. A 85, 043627 (2012).

[29] M. Karl and T. Gasenzer, New J. Phys. 19, 093014 (2017).

[30] D. V. Freilich, D. M. Bianchi, A. M. Kaufman, T. K. Langin, and D. S. Hall, Science 329, 1182 (2010).

[31] T. W. Neely, E. C. Samson, A. S. Bradley, M. J. Davis, and B. P. Anderson, Phys. Rev. Lett. 104, 160401 (2010).

[32] S. Middelkamp, P. J. Torres, P. G. Kevrekidis, D. J. Frantzeskakis, R. Carretero-González, P. Schmelcher, D. V. Freilich, and D. S. Hall, Phys. Rev. A 84, 011605 (2011).

[33] W. J. Kwon, S. W. Seo, and Y. Shin, Phys. Rev. A 92, 033613 (2015).

[34] R. Navarro, R. Carretero-González, P. J. Torres, P. G. Kevrekidis, D. J. Frantzeskakis, M. W. Ray, E. Altuntaş, and D. S. Hall, Phys. Rev. Lett. 110, 225301 (2013).

[35] W. J. Kwon, J. H. Kim, S. W. Seo, and Y. Shin, Phys. Rev. Lett. 117, 245301 (2016).

[36] E. A. L. Henn, J. A. Seman, G. Roati, K. M. F. Magalhães, and V. S. Bagnato, Phys. Rev. Lett. 103, 045301 (2009).

[37] P. E. S. Tavares, A. R. Fritsch, G. D. Telles, M. S. Hussein, F. Impens, R. Kaiser, and V. S. Bagnato, arXiv:1606.01589.

[38] G. W. Stagg, A. J. Allen, N. G. Parker, and C. F. Barenghi, Phys. Rev. A 91, 013612 (2015).

[39] A. Powis, S. Sammut, and T. Simula, Phys. Rev. Lett. 113, 165303 (2014).

[40] K. E. Wilson, Z. L. Newman, J. D. Lowney, and B. P. Anderson, Phys. Rev. A 91, 023621 (2015).

[41] See Supplemental Material at http://link.aps.org/ supplemental/10.1103/PhysRevLett.120.034504 for further details, which includes Refs. [20,31,42].

[42] C. Raman, M. Köhl, R. Onofrio, D. S. Durfee, C. E. Kuklewicz, Z. Hadzibabic, and W. Ketterle, Phys. Rev. Lett. 83, 2502 (1999).

[43] Y. B. Pointin and T. S. Lundgren, Phys. Fluids 19, 1459 (1976).

[44] R. N. Valani, A. J. Groszek, and T. P. Simula, arXiv: 1612.02930

[45] V. L. Berezinskii, J. Exp. Theor. Phys. 32, 493 (1971).

[46] V. L. Berezinskii, J. Exp. Theor. Phys. 34, 610 (1972).

[47] J. M. Kosterlitz and D. J. Thouless, J. Phys. C 6, 1181 (1973).

[48] Z. Hadzibabic, P. Krüger, M. Cheneau, B. Battelier, and J. Dalibard, Nature (London) 441, 1118 (2006).

[49] P. Cladé, C. Ryu, A. Ramanathan, K. Helmerson, and W. D. Phillips, Phys. Rev. Lett. 102, 170401 (2009).

[50] X. Yu, T. P. Billam, J. Nian, M. T. Reeves, and A. S. Bradley, Phys. Rev. A 94, 023602 (2016). 